\title{
Identification of Unknown Parameters of Partially Observed Discrete-time Stochastic Systems by Using Pseudomeasurements*
}

\author{
Akio TANIKAWA ${ }^{\dagger}$ and Yuichi SAWADA ${ }^{\ddagger}$
}

\begin{abstract}
We consider partially observed discrete-time linear stochastic systems and assume that some entries of the system matrices are unknown. We propose a new method which identifies these unknown entries and the state vectors of these systems simultaneously. The key idea of the proposed method is utilization of the pseudomeasurement which is a fictitious and additional observation process on the unknown entries and will be modified so as to work for the partially observed systems. Augmenting the pseudomeasurement with the original observation process, we derive the new identification method by applying the extended Kalman filter. The proposed method is consistent with the conventional method (without pseudomeasurement).
\end{abstract}

\section{Introduction}

Identification of stochastic dynamical systems from input and output data is a fundamental and important problem, as recognized as a kind of inverse problems. Over the last two decades, one of the most popular methods to identify these systems has been the subspace-based identification method and a considerable number of research papers using this method have been published (see $[8,19]$ ). To fix the idea of the subspace-based method, consider the linear stochastic system described by a couple of equations

$$
\begin{aligned}
x(t+1) & =A x(t)+B u(t)+w(t), \\
y(t) & =C x(t)+v(t),
\end{aligned}
$$

where $w(t)$ and $v(t)$ are random noise sequences. In the subspace-based method, identification of the system matrices $(A, B, C)$ is conducted from the input and output data $\{u(t), y(t)\}$ within a similarity transformation such as $\left(A_{T}, B_{T}, C_{T}\right)$ where $A_{T}=T^{-1} A T, B_{T}$ $=T^{-1} B, C_{T}=C T$ and $T$ is a transformation matrix. Thus, the matrices $(A, B, C)$ cannot be identified individually by the subspace-based method because the transformation matrix $T$ cannot be determined.

In this paper, we assume that some entries of the system matrix $A$ are unknown and denote the known part (resp. the unknown part) of $A$ by $A_{k}$ (resp. $A_{u}$ )

* Manuscript Received Date: May 19, 2014

$\dagger$ Faculty of Information Science and Technology, Osaka Institute of Technology; Kitayama, Hirakata-shi, Osaka 573-0196, JAPAN

₹ Department of Mechanical and System Engineering, Kyoto Institute of Technology; Matsugasaki, Sakyo, Kyoto 606-8585, JAPAN

Key Words: system identification, pseudomeasurement, extended Kalman filter. (see Section 3 for the detail). We propose a new iterative method which identifies those unknown parameters and the state vector of the system simultaneously by making use of $A_{k}$ explicitly. By the subspace-based method, we can have an estimate of the matrix $A$ from the estimate of $\left(A_{T}, B_{T}, C_{T}\right)$, but it is impossible to utilize the information about the known part $A_{k}$ of $A$ explicitly. However, in Section 5, we will propose the iterative method which identifies both the states of the system and the unknown part $A_{u}$ of $A$ by using $A_{k}$ explicitly. This is one of the advantages of the the proposed method in this paper over the subspacebased method.

The main idea of the proposed method is to use pseudomeasurement which is a fictitious observation process on the unknown entries of the stochastic dynamical systems. Firstly, the pseudomeasurement was utilized for various tracking problems by several researchers (see e.g., $[1,15,16,20]$ ). Later, Ohsumi and his colleagues applied the idea of pseudomeasurement to tracking problems of maneuvering ships ([13,14]) and some identification problems in environmental pollution problems $([11,12])$, and also to identification problems of unknown exogenous inputs and related quantities $([5,6,7,9,10])$.

Recently, Kameyama and Ohsumi applied it to identification problems of unknown entries of the system matrices $([2,3])$. In our previous paper [18], we studied the same kind of identification problems for discrete-time linear stochastic systems while Ohsumi and his colleagues dealt with continuous-time linear stochastic systems. We proposed a new identification method in[18] only for the case where the dimension of the observation process is the same as that of the dynamical system and the coefficient matrix $H$ is nonsingular (see Section 2 for the detail). However, 
in this paper, we consider the case where the dimension of the observation process is smaller than that of the dynamical system. Namely, we investigate a new identification method for partially observed (discretetime) linear stochastic systems.

After introducing the pseudomeasurement for the given stochastic system, we augment it with the original observation process and derive the new identification method by applying the extended Kalman filter. Due to the additional observation process data (i.e., pseudomeasurement), we can expect better performance of the new identification method than that of the conventional approach. It can be seen in the simulation results in Section 6 .

\section{Problem Statement}

Consider the discrete-time linear stochastic system for $\mathrm{t}=0,1,2, \ldots$ :

$$
\begin{aligned}
x(t+1) & =A x(t)+C u(t)+G w(t), \\
y(t) & =H x(t)+S v(t),
\end{aligned}
$$

where $x(t) \in \mathbb{R}^{n}, y(t) \in \mathbb{R}^{n}$ and $u(t) \in \mathbb{R}^{\ell}$ denote the state vector of the system, the observation vector and the input vector, respectively; $A \in \mathbb{R}^{n \times n}$ (i.e., a set of $n \times n$ matrices), $C \in \mathbb{R}^{n \times \ell}, G \in \mathbb{R}^{n \times d_{1}}, H \in$ $\mathbb{R}^{m \times n}$ and $S \in \mathbb{R}^{m \times d_{2}}$. Here, we assume that the matrices $C, G, H$ and $S$ are known but the matrix $A$ is partially unknown (see Section $\mathbf{3}$ for the detail). Moreover, $w(t)\left(\in \mathbb{R}^{d_{1}}\right)$ and $v(t)\left(\in \mathbb{R}^{d_{2}}\right)$ are independent zero mean white Gaussian noise sequences with the covariance matrices $Q$ and $R$, respectively, i.e., $\mathcal{E}\left\{w(t) w(t)^{T}\right\}=Q$ and $\mathcal{E}\left\{v(t) v(t)^{T}\right\}=R$, where $\mathcal{E}\{\cdot\}$ denotes the mathematical expectation operator and $T$ denotes transposition of a matrix. In the previous paper[18], we dealt with the case where $m=n$ holds and the matrix $H$ is nonsingular, whereas we here assume $m<n$ and that the rank of $H$ is $m$. The pair $(A, H)$ is assumed tacitly to be observable. Let $Y_{t}$ be the $\sigma$-field $\sigma\{y(s), 0 \leq s \leq t\}$ generated by the observation data $y(s)$ up to the time $t$.

\section{Pseudomeasurement Approach}

Let $A=\left(a_{i j}\right)$ be an $n \times n$ matrix in which some of the elements are assumed to be unknown. We define the $n \times n$ matrices $A_{k}$ and $A_{u}$, the known part of $A$ and the unknown part of $A$ respectively as follows:

$$
\begin{array}{lll}
\left(A_{k}\right)_{i j}=a_{i j}, & \left(A_{u}\right)_{i j}=0 & \text { if } a_{i j} \text { is known, } \\
\left(A_{k}\right)_{i j}=0, & \left(A_{u}\right)_{i j}=a_{i j} & \text { if } a_{i j} \text { is unknown, }
\end{array}
$$

where $(B)_{i j}$ denotes the $(i, j)$-element of the matrix $B$. Then, we immediately have

$$
A=A_{k}+A_{u} .
$$

Assuming that the number of unknown entries is $p$ we define the $p$-dimensional vector $a$ by rearranging $p$ unknown entries $\left\{a_{i j}\right\}$ in the order from upper to lower in the first column and so on. We also denote the matrix $A_{u}$ as $A_{u}(a)$ to indicate the unknown parameter vector $a$ explicitly in the following.
It is easy to find the $n \times p$ matrix $X(t)$ satisfying

$$
A_{u}(a) x(t)=X(t) a,
$$

where $X(t)$ can be written as $X(t)=\sum_{i=1}^{n} x_{i}(t) M_{i}$ for some $n \times p$ matrices $M_{1}, M_{2}, \cdots, M_{n}$ depending on $A_{u}(a)$ (see[3] for the detail). We also have

$$
X(t)=M_{0}\left(x(t) \otimes I_{p}\right),
$$

where $M_{0}$ denotes the $n \times n p$ matrix $\left[M_{1} M_{2} \cdots M_{n}\right]$. Here, the symbol $\otimes$ denotes the Kronecker's product of matrices and $I_{p}$ denotes the $p \times p$ identity matrix.

Denoting the pseudoinverse of $H$ by $H^{\dagger}$, we define the $n \times n$ matrix $L$ by $L:=H^{\dagger} H$ and assume the following condition.

[Condition A] The following condition holds:

$D(d):=L M_{0}\left[(I-L) d \otimes I_{p}\right]=O$ for any $d\left(\in \mathbb{R}^{n}\right)$.(8)

From (6)-(7), we can rewrite (3) as

$$
\begin{aligned}
x(t+1)=A_{k} x(t) & +M_{0}\left(x(t) \otimes I_{p}\right) a \\
& +C u(t)+G w(t) .
\end{aligned}
$$

We now seek a relation between the unknown vector $a$ and the observation process $\{y(t)\}$. Firstly, it immediately follows from (4) that

$$
L x(t)=H^{\dagger} H x(t)=H^{\dagger} y(t)-H^{\dagger} S v(t) .
$$

Defining the matrix $B$ by

$$
B:=L A_{k}(I-L),
$$

we have

$$
\begin{aligned}
& H^{\dagger} y(t+1)=H^{\dagger} H x(t+1)+H^{\dagger} S v(t+1) \\
& =L\left\{A_{k} x(t)+M_{0}\left(x(t) \otimes I_{p}\right) a+C u(t)+G w(t)\right\} \\
& \quad+H^{\dagger} S v(t+1) \\
& =L A_{k} H^{\dagger} y(t)+B x(t)+L M_{0}\left(H^{\dagger} y(t) \otimes I_{p}\right) a \\
& \quad+L C u(t)+L G w(t)-L A_{k} H^{\dagger} S v(t) \\
& \quad-L M_{0}\left(H^{\dagger} S v(t) \otimes I_{p}\right) a+H^{\dagger} S v(t+1)
\end{aligned}
$$

from (9), (10) and Condition A.

We here define the new process

$$
y_{p}(t)=H^{\dagger} y(t+1)-L A_{k} H^{\dagger} y(t)-L C u(t)
$$

which is obviously a simple known function of the input vector $u(t)$ and the observation vectors $y(t)$ and $y(t+1)$. Defining the new matrix

$$
H_{p}(t)=L M_{0}\left(H^{\dagger} y(t) \otimes I_{p}\right),
$$

we have the equality

$$
y_{p}(t)=B x(t)+H_{p}(t) a+(\text { noise terms }),
$$

due to (12)-(13).

Since the noise terms in (15) are a linear combination of the mutually independent Gaussian white noises $w(t), v(t)$ and $v(t+1)$ and $a$ is a constant vector, they can be written as a single Gaussian noise. 
Thus, the equality (15) can be rewritten as

$$
y_{p}(t)=B x(t)+H_{p}(t) a(t)+S_{p} v_{p}(t),
$$

with $S_{p} \in \mathbb{R}^{n \times d_{4}}$, where $\left\{v_{p}(t)\left(\in \mathbb{R}^{d_{4}}\right)\right\}$ is a zero-mean Gaussian (but non-white) noise sequence with a covariance matrix $R_{p}$ which has a correlation with the previously given Gaussian white noise sequences $\{v(t)\}$ and $\{w(t)\}$. Thus, $\left\{y_{p}(t)\right\}$ can be regarded as the observation process of the unknown vector $a$. We discuss when the pair $\left(A_{u}, H\right)$ of the matrices satisfy Condition $\mathrm{A}$ in Appendix. We will present the modified pseudomeasurement method which can be applied to the case where Condition A does not hold in the forthcoming paper[17].

\section{Augmented System}

To identify the unknown vector $a$, we usually consider it as a function $a(t)$ of $t$ and treat it as a random vector-valued process

$$
a(t+1)=a(t)+G_{a} w_{a}(t)
$$

by allowing some ambiguity. Here, $\left\{w_{a}(t)\left(\in \mathbb{R}^{d_{3}}\right)\right\}$ is a newly introduced zero-mean Gaussian white noise sequence with covariance matrix $Q_{a}$ which is independent with previously given random sequences. Notice that the matrices $G_{a}$ and $Q_{a}$ can be preassigned by the users. Actually, we may choose $G_{a}=O$ if we need no ambiguity in (17).

Let us introduce a new state vector defined by $z(t):=\left[x(t)^{T}, a(t)^{T}\right]^{T}$. The following augmented system can be obtained from (9) and (17):

$$
z(t+1)=f(z(t))+C_{0} u(t)+G_{0} w_{0}(t),
$$

where

$$
\begin{aligned}
& f(z(t))=\left(f_{1}(z(t)), f_{2}(z(t)), \ldots, f_{n+p}(z(t))\right)^{T} \\
& =\left[\begin{array}{cc}
A_{k} & X(t) \\
O & I
\end{array}\right] z(t) \in \mathbb{R}^{n+p}, \\
& C_{0}=\left[\begin{array}{l}
C \\
O
\end{array}\right] \in \mathbb{R}^{(n+p) \times \ell}, \\
& G_{0}=\left[\begin{array}{cc}
G & O \\
O & G_{a}
\end{array}\right] \in \mathbb{R}^{(n+p) \times\left(d_{1}+d_{3}\right)}, \\
& w_{0}(t)=\left[\begin{array}{c}
w(t) \\
w_{a}(t)
\end{array}\right] \in \mathbb{R}^{d_{1}+d_{3}} .
\end{aligned}
$$

Defining the new $(m+n)$-dimensional vector $y_{0}(t)$ $:=\left[y(t)^{T}, y_{p}(t)^{T}\right]^{T}$ and the new $\left(d_{2}+d_{4}\right)$-dimensional vector $v_{0}(t):=\left[v(t)^{T}, v_{p}(t)^{T}\right]^{T}$, we can obtain the augmented observation process

$$
y_{0}(t)=H_{0}(t) z(t)+S_{0} v_{0}(t)
$$

from (4) and (16), where we used the notation

$$
H_{0}(t)=\left[\begin{array}{cc}
H & O \\
B & H_{p}(t)
\end{array}\right] \in \mathbb{R}^{(m+n) \times(n+p)}
$$

and

$$
S_{0}=\left[\begin{array}{cc}
S & O \\
O & S_{p}
\end{array}\right] \in \mathbb{R}^{(m+n) \times\left(d_{2}+d_{4}\right)} .
$$

\section{Extended Kalman Filter}

To estimate the state vector $x(t)$ and identify the unknown entries $a$ simultaneously, we propose to apply the extended Kalman filter (EKF) to the augmented nonlinear system (18) with (19) although the noise sequence $\left\{v_{p}(t)\right\}$ is not white and has a correlation with the previously given Gaussian white noise sequences $\{w(t)\}$ and $\{v(t)\}$. (We will consider a modification of the EKF-based method in a forthcoming paper when the correlation between these noises are not negligible.) Thus, supposing that the estimate $\widehat{z}(t \mid t)$ of $z(t)$ has been obtained, we use the linear approximation of $f$ around $\widehat{z}(t \mid t)$

$$
\begin{aligned}
f(z(t))=f(\widehat{z}(t \mid t)) & +\widehat{F}_{t}(z(t)-\widehat{z}(t \mid t)) \\
& +(\text { higher order terms }),
\end{aligned}
$$

and obtain the linearized system of (18)

$$
\begin{aligned}
z(t+1)=\widehat{F}_{t} z(t)+ & C_{0} u(t)+G_{0} w_{0}(t) \\
& +f(\widehat{z}(t \mid t))-\widehat{F}_{t} \widehat{z}(t \mid t),
\end{aligned}
$$

where

$$
\begin{aligned}
& \widehat{F}_{t}=\left(\frac{\partial f(z)}{\partial z^{T}}\right)_{z=\widehat{z}(t \mid t)}=\left[\left(\frac{\partial f^{T}(z)}{\partial z}\right)_{z=\widehat{z}(t \mid t)}\right]^{T} \\
& =\left[\begin{array}{cccc}
\frac{\partial f_{1}(\widehat{z})}{\partial z_{1}} & \frac{\partial f_{1}(\widehat{z})}{\partial z_{2}} & \cdots & \frac{\partial f_{1}(\widehat{z})}{\partial z_{n+p}} \\
\vdots & \vdots & \ddots & \vdots \\
\frac{\partial f_{n+p}(\widehat{z})}{\partial z_{1}} & \frac{\partial f_{n+p}(\widehat{z})}{\partial z_{2}} & \cdots & \frac{\partial f_{n+p}(\widehat{z})}{\partial z_{n+p}}
\end{array}\right] .
\end{aligned}
$$

Applying the Kalman filter to the linearized system (21) with (19), we have the following results. Here, $\widehat{z}(t+\mid t)$ and $\widehat{z}(t+1 \mid t+1)$ denote the optimal estimates of $z(t+1)$ given by the Kalman filter for the linearized system (21) with (19) respectively with respect to $Y_{t}$ and $Y_{t+1}$. We also define the matrices $P(t \mid t)$ and $P(t+1 \mid t)$ as follows:

and

$$
\begin{gathered}
P(t \mid t)=\mathcal{E}\left[\{z(t)-\widehat{z}(t \mid t)\}\{z(t)-\widehat{z}(t \mid t)\}^{T}\right] \\
P(t+1 \mid t)=\mathcal{E}[\{z(t+1)-\widehat{z}(t+1 \mid t)\} \\
\left.\{z(t+1)-\widehat{z}(t+1 \mid t)\}^{T}\right] .
\end{gathered}
$$

[Proposition 1] The optimal estimates $\widehat{z}(t+1 \mid t)$ and $\widehat{z}(t+1 \mid t+1)$ obtained by the Kalman filter applied to the linearized system (21) with (19) have the following form:

$$
\begin{aligned}
& \widehat{z}(t+1 \mid t)=f(\widehat{z}(t \mid t))+C_{0} u(t), \\
& \widehat{z}(t+1 \mid t+1)=\widehat{z}(t+1 \mid t) \\
& \quad+K(t+1)\left[y_{0}(t+1)-H_{0}(t+1) \widehat{z}(t+1 \mid t)\right],
\end{aligned}
$$

where 


$$
\begin{aligned}
& K(t+1)=P(t+1 \mid t) H_{0}^{T}(t+1) \\
& \times\left[H_{0}(t+1) P(t+1 \mid t) H_{0}^{T}(t+1)+S_{0} R_{0} S_{0}^{T}\right]^{-1}, \\
& R_{0}=\left[\begin{array}{cc}
R & O \\
O & R_{p}
\end{array}\right] \in \mathbb{R}^{\left(d_{2}+d_{4}\right) \times\left(d_{2}+d_{4}\right)} .
\end{aligned}
$$

Here, $\widehat{z}(0 \mid-1)=\bar{z}_{0}$ is the initial estimate and $P(0 \mid-1)$ is chosen to be $c I$ for some $c(>0)$. Moreover, the matrices $P(t+1 \mid t)$ and $P(t \mid t)$ satisfy the recursive relations

$$
P(t+1 \mid t)=\widehat{F}_{t} P(t \mid t) \widehat{F}_{t}^{T}+G_{0} Q_{0} G_{0}^{T}
$$

and

$$
P(t \mid t)=P(t \mid t-1)-K(t) H_{0}(t) P(t \mid t-1) .
$$

respectively, where

$$
Q_{0}=\left[\begin{array}{cc}
Q & O \\
O & Q_{p}
\end{array}\right] \in \mathbb{R}^{\left(d_{1}+d_{3}\right) \times\left(d_{1}+d_{3}\right)} .
$$

(Proof) Applying the Kalman filter to the linear system (21) with (19), we have

$$
\begin{aligned}
\widehat{z}(t+1 \mid t) & =\widehat{F}_{t} \widehat{z}(t \mid t)+C_{0} u(t)+f(\widehat{z}(t \mid t))-\widehat{F}_{t} \widehat{z}(t \mid t) \\
& =f(\widehat{z}(t \mid t))+C_{0} u(t)
\end{aligned}
$$

and

$$
\begin{aligned}
\widehat{z}(t+1 \mid t+1) & =\widehat{z}(t+1 \mid t) \\
& +K(t+1)\left[y_{0}(t+1)-H_{0}(t+1) \widehat{z}(t+1 \mid t)\right] .
\end{aligned}
$$

The rest of the results can be obtained by the standard argument of the Kalman filter.

[Proposition 2] The equalities (24) and (25) can be written explicitly as follows:

$$
\begin{aligned}
& {\left[\begin{array}{l}
\widehat{x}(t+1 \mid t) \\
\widehat{a}(t+1 \mid t)
\end{array}\right]} \\
& \quad=\left[\begin{array}{c}
A_{k} \widehat{x}(t \mid t)+M_{0}\left(\widehat{x}(t \mid t) \otimes I_{p}\right) \widehat{a}(t \mid t)+C u(t) \\
\widehat{a}(t \mid t)
\end{array}\right]
\end{aligned}
$$

and

$$
\begin{aligned}
& {\left[\begin{array}{l}
\widehat{x}(t+1 \mid t+1) \\
\widehat{a}(t+1 \mid t+1)
\end{array}\right]=\left[\begin{array}{l}
\widehat{x}(t+1 \mid t) \\
\widehat{a}(t+1 \mid t)
\end{array}\right]+K(t+1)} \\
& \times\left[\begin{array}{r}
y(t+1)-H \widehat{x}(t+1 \mid t) \\
y_{p}(t+1)-B \widehat{x}(t+1 \mid t)-H_{p}(t+1) \widehat{a}(t+1 \mid t)
\end{array}\right] .
\end{aligned}
$$

(Proof) Since

$$
\begin{aligned}
f(z(t)) & =\left[\begin{array}{cc}
A_{k} & X(t) \\
O & I
\end{array}\right]\left[\begin{array}{l}
x(t) \\
a(t)
\end{array}\right] \\
& =\left[\begin{array}{c}
\left\{A_{k}+A_{u}(a(t))\right\} x(t) \\
a(t)
\end{array}\right],
\end{aligned}
$$

we have

$$
\begin{aligned}
f(\widehat{z}(t \mid t)) & =\left[\begin{array}{c}
\left\{A_{k}+A_{u}(\widehat{a}(t \mid t))\right\} \widehat{x}(t \mid t) \\
\widehat{a}(t \mid t)
\end{array}\right] \\
& =\left[\begin{array}{c}
\widehat{A}(t \mid t) \widehat{x}(t \mid t) \\
\widehat{a}(t \mid t)
\end{array}\right],
\end{aligned}
$$

where $\widehat{A}(t \mid t)=A_{k}+A_{u}(\widehat{a}(t \mid t))$. Thus we have (29). The equality (30) immediately follows from (25).

[Remark 3] We note that

$$
\begin{aligned}
& \frac{\partial f^{T}(z)}{\partial z}=\frac{\partial}{\partial z}\left[x^{T}\left\{A_{k}+A_{u}(a)\right\}^{T} \quad a^{T}\right] \\
& =\left[\begin{array}{cc}
\frac{\partial}{\partial x} x^{T}\left\{A_{k}+A_{u}(a)\right\}^{T} & \frac{\partial}{\partial x} a^{T} \\
\frac{\partial}{\partial a} x^{T}\left\{A_{k}+A_{u}(a)\right\}^{T} & \frac{\partial}{\partial a} a^{T}
\end{array}\right] \\
& =\left[\begin{array}{cc}
\left\{A_{k}+A_{u}(a)\right\}^{T} & 0 \\
X^{T} & I
\end{array}\right]
\end{aligned}
$$

holds, where we used the equality

$$
\frac{\partial}{\partial a} x^{T} A_{u}^{T}(a)=\frac{\partial}{\partial a} a^{T} X^{T}=X^{T} .
$$

Thus we have

$$
\begin{aligned}
\widehat{F}_{t} & =\left(\frac{\partial f(z)}{\partial z^{T}}\right)_{z=\widehat{z}(t \mid t)} \\
& =\left[\begin{array}{cc}
A_{k}+A_{u}(a) & X \\
O & I
\end{array}\right]_{x=\widehat{x}, a=\widehat{a}} \\
& =\left[\begin{array}{cc}
\widehat{A}(t \mid t) & \widehat{X}(t \mid t) \\
O & I
\end{array}\right],
\end{aligned}
$$

where

$$
\widehat{X}(t \mid t)=\left.X(t)\right|_{x=\widehat{x}}=\sum_{i=1}^{n} \widehat{x}_{i}(t \mid t) M_{i} .
$$

We state one advantage of the proposed method here. By choosing $H_{0}=\left[\begin{array}{ll}H & O_{m \times p}\end{array}\right]$ without using $y_{p}(t)$ and $H_{p}(t)$, the proposed method reduces to the conventional method (without the pseudomeasurement). Thus, our method is consistent with the conventional method, and so we have the choice of the proposed method (by utilizing (20)) or the conventional method (by choosing $H_{0}=\left[\begin{array}{ll}H & O_{m \times p}\end{array}\right]$ ). This is an advantage of the proposed method in practice.

\section{Numerical Simulation}

We applied the new identification method via pseudomeasurement approach and the conventional identification method (without pseudomeasurement) to a simple example and compared the results. We consider the single-input two-output three-dimensional linear system with

$$
\begin{aligned}
& A=\left[\begin{array}{lll}
0 & 0 & d_{1} \\
1 & 0 & d_{2} \\
0 & 1 & a
\end{array}\right], C=\left[\begin{array}{l}
0 \\
0 \\
1
\end{array}\right], G=\left[\begin{array}{ll}
0 & 1 \\
0 & 0 \\
1 & 0
\end{array}\right], \\
& H=\left[\begin{array}{lll}
0 & 1 & 0 \\
0 & 0 & 1
\end{array}\right], S=I_{2}, \quad Q=I_{2}, \quad R=0.01 I_{2},
\end{aligned}
$$

where the elements $d_{1}$ and $d_{2}$ of $A$ are known and chosen as $d_{1}=0.7425$ and $d_{2}=-2.4660$ respectively. On the other hand, the element $a$ is unknown. We assign the true parameter $a$ as follows: $a=2.7200$ (see page 127 of $[4])$. In the simulation, the initial state of system (3) is given by $x(0)=[1,1,1]^{T}$, and the known input is given by $u(t) \equiv 1$. We applied the identification method via pseudomeasurement approach 
starting from the initial estimates as follows: $\widehat{x}(0 \mid-$ $1)=[0,0,0]^{T}, \widehat{a}(0 \mid-1)=5, P(0 \mid-1)=0.9 \times I_{4}$. Userdefined parameters are given by $G_{a}=I_{1}, S_{p}=I_{3}, Q_{a}=$ $I_{1}$ and $R_{p}=10 I_{3}$.

The simulation results via pseudomeasurement approach are shown as Simulation 1 in Figs. 1-5. While Fig. 1 depicts the trajectory of stochastic system (3), the state estimate $\widehat{x}(t \mid t)$ and the identified parameter $\widehat{a}(t \mid t)$ via pseudomeasurement are shown in Fig. 2 and Fig. 3, respectively. The trajectories of the diagonal elements $(1,1)-(3,3)$ (resp. $(4,4))$ of the estimation error covariance matrix $P(t \mid t)$ are given in Fig. 4 (resp. Fig. 5). For comparison, we also show the simulation results by the conventional identification method using the extended Kalman filter (without pseudomeasurement) as Simulation 2 in Figs. 6-10, i.e., Fig. 6 for the state trajectory $x(t)$ of (3), Fig. 7 for the state estimate $\widehat{x}(t \mid t)$ of $x(t)$ and Fig. 8 for the identified parameter $\widehat{a}(t \mid t)$, respectively. Similarly to Figs. 4 and 5 , the trajectories of the diagonal elements of $P(t \mid t)$ are given in Figs. 9 and 10 for the conventional identification method without pseudomeasurement .

In view of Figs. 1, 2, 4 and Figs. 6, 7, 9, there is no big difference between the two methods on the state estimation. However, we can see the difference between Figs. 3, 5 and Figs. 8, 10. Namely, the identified parameter $\widehat{a}(t \mid t)$ and the $a$-part of the covariance matrix $P(t \mid t)$ via pseudomeasurement in Figs. 3 and 5 are better than those by the conventional method in Figs. 8 and 10.

The estimation errors $\|\widehat{a}(t \mid t)-a\|$ of the unknown parameter $a$ by the two methods were computed respectively for 20 numerical experiments. The mean

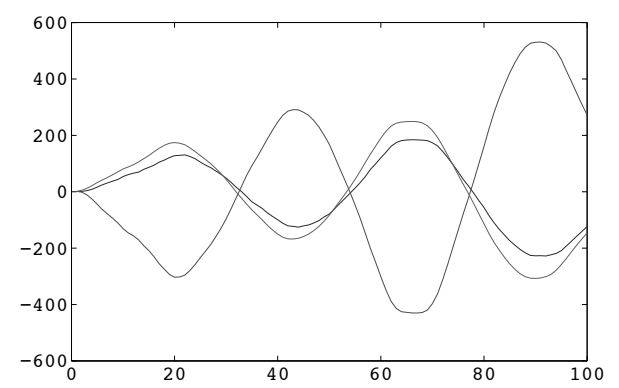

Fig. 1 State trajectory of the linear stochastic system (Simulation 1)

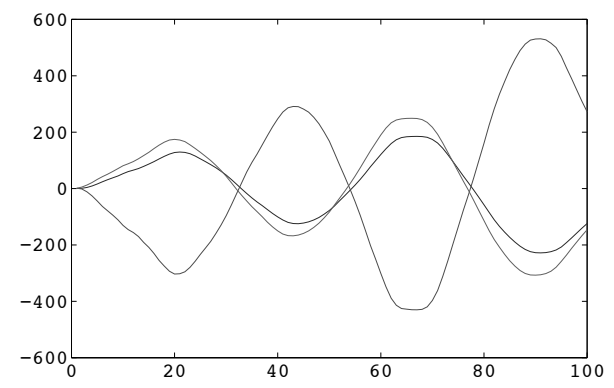

Fig. 2 State estimation via pseudomeasurement (Simulation 1)

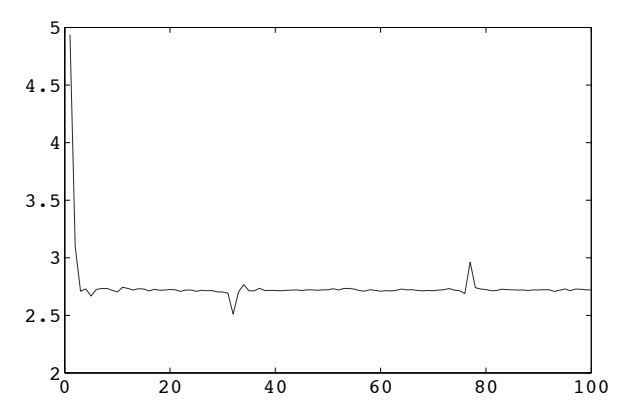

Fig. 3 Identified process of $a$ via pseudomeasurement (Simulation 1)

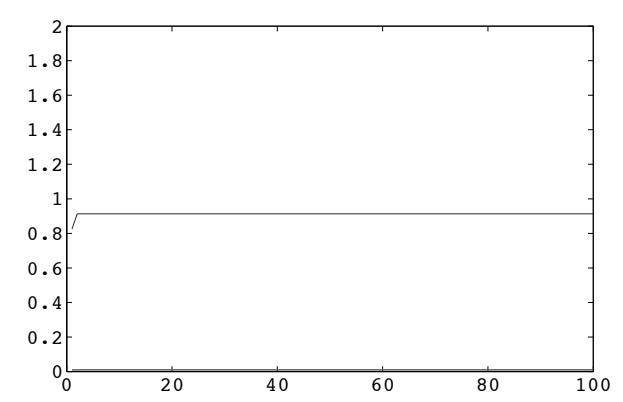

Fig. $4 \quad x$-part of $P(t \mid t)$ via pseudomeasurement (Simulation 1)

and the standard deviation of the average of the estimation error $\|\widehat{a}(t \mid t)-a\|$ for $10<t \leq 100$ by the first method (with the pseudomeasurement) were 0.01411 and 0.00644 respectively. On the other hand, those by the second method (without the pseudomeasurement) were 0.02242 and 0.00983 respectively. Thus, the mean and the standard deviation of the error became less than two thirds respectively due to the utilization of the pseudomeasurement.

\section{Conclusions}

We consider partially observed discrete-time linear stochastic systems and assume that some entries of the system matrices are unknown. We present a new method which identifies unknown entries of the system matrix $A$ and the state vector of the system simultaneously. The key idea of the proposed method is utilization of the pseudomeasurement which is a fictitious and additional observation process on the unknown entries and was modified so as to work for the partially observed systems. The proposed method is consistent with the conventional method (without pseudomeasurement) and so they can easily be unified to be a single iterative process for simultaneous identification and state estimation by switching the coefficient matrix of the augmented observation process. This is an advantage of the proposed method in practice. The simulation results show the effectiveness of the proposed method.

\section{Acknowledgements}

Part of this research was supported by the Japan Society for Promotion of Science (JSPS) under Grant- 
in Aid for Scientific Research (C)-22540158 and (C)25400149. The authors wish to thank the referees for their helpful comments in the improvement of this paper. The first author wishes to thank Professor Takashi Saito for his kind assistance in preparing the manuscript.

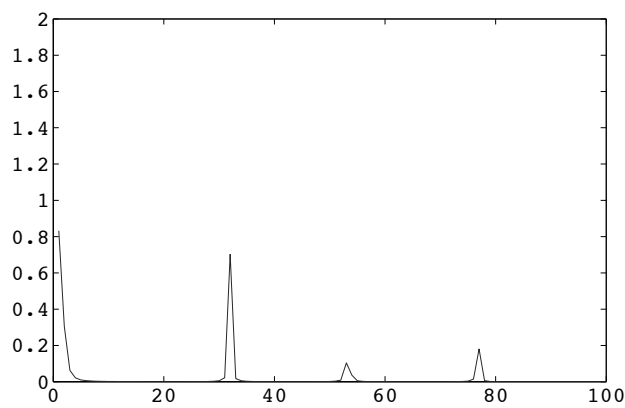

Fig. $5 \quad a$-part of $P(t \mid t)$ via pseudomeasurement (Simulation 1)

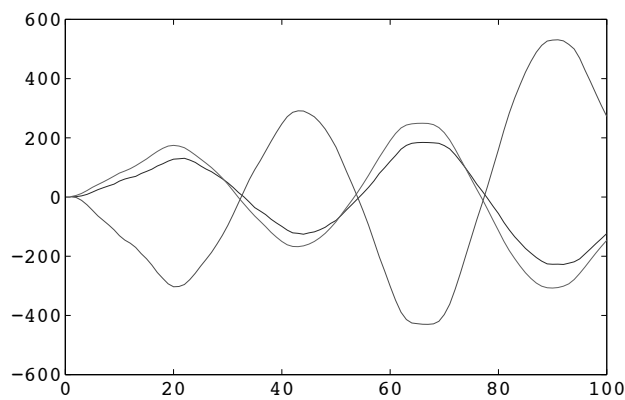

Fig. 6 State trajectory of the linear stochastic system (Simulation 2)

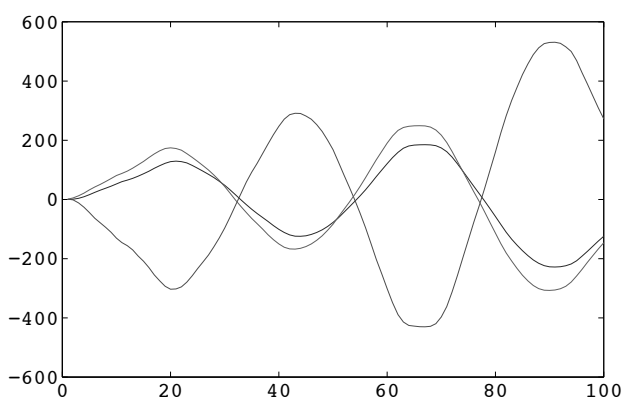

Fig. 7 State estimation for without pseudomeasurement (Simulation 2)

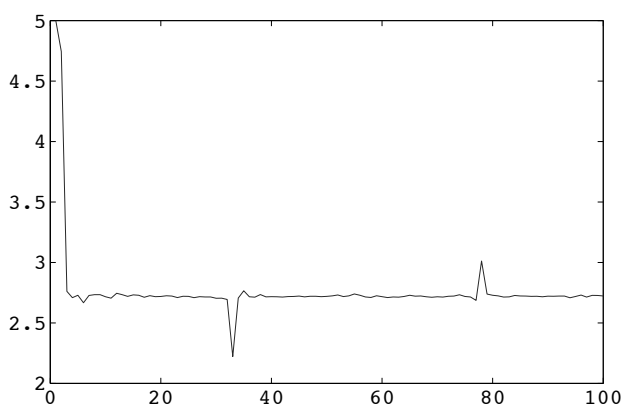

Fig. 8 Identified process of $a$ without pseudomeasurement (Simulation 2)

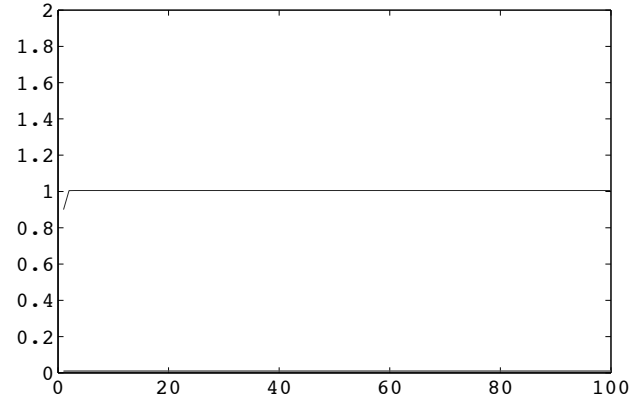

Fig. $9 x$-part of $P(t \mid t)$ without pseudomeasurement (Simulation 2)

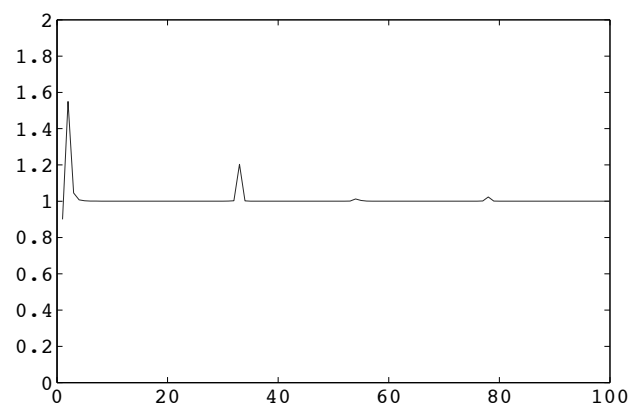

Fig. $10 \quad a$-part of $P(t \mid t)$ without pseudomeasurement (Simulation 2)

\section{References}

[1] A. T. Alouani and W. D. Bair: Use of a kinematic constraint in tracking constant speed, maneuvering target; Proc. of the 30th Conf. on Decision and Control, pp. 2055-2058 (1991)

[2] K. Kameyama and A. Ohsumi: Identification of unknown parameters of linear systems from noisy observation data using pseudomeasurement approach; Proc. of the SICE Annual Conference on Control Systems, CD-ROM 185-2-3 (2010)

[3] K. Kameyama and A. Ohsumi: Identification of partially unknown system matrix from noisy observation data via pseudomeasurement approach; Proc. of the 42nd ISCIE Int. Symp. on Stochastic System Theory and Its Applications, pp. 27-32 (2010)

[4] T. Katayama: Applied Kalman Filters, 2nd Edition, Asakura-Shoten (2000) (in Japanese)

[5] T. Kimura, A. Ohsumi and M. Kono: Identification of unknown exogenous input to stochastic linear systems using pseudomeasurements; Trans. ISCIE, Vol. 21, No. 12 , pp. 390-399 (2008) (in Japanese)

[6] T. Kimura, A. Ohsumi and M. Kono: Observer-based identification of unknown exogenous input via pseudomeasurement approach; SICE J. of Control, Measurement and System Integration, Vol. 2, No. 3, pp. 184-191 (2009)

[7] A. Ohsumi: An outlook on the use of pseudomeasurement in system identification; Proc. of the 37th SICE Symp. on Control Systems, pp. 91-96 (2008)

[8] A. Ohsumi, K. Kameyama and K.-I Yamaguchi: Subspace identification for continuous-time stochastic systems via distribution-based approach; Automatica, Vol. 38, No. 2, pp.63-79 (2002)

[9] A. Ohsumi, T. Kimura and M. Kono: Kalman filter- 
based identification of unknown exogenous input of stochastic linear systems via pseudomeasurement approach; Int. J. of Innovative Computing, Information and Control, Vol. 5, No. 1, pp. 1-17 (2009)

[10] A. Ohsumi, T. Kimura and M. Kono: A novel approach to the identification of exogenous input of stochastic systems using pseudomeasurement; Proc. of IFAC Symp. SYSID 2009, pp. 296-301 (2009)

[11] A. Ohsumi, S. Komiyama, K. Kashiwagi, M. Watanabe and T. Takatsu: Detection of polluted load and identification of its discharged location and magnitude for polluted river; Int. J. of Innovative Computing, Information and Control, Vol. 4, No. 1, pp. 63-77 (2008)

[12] A. Ohsumi, S. Komiyama, M. Watanabe, K. Kashiwagi and T. Takatsu: Identification of polluted load and its discharged location and estimation of water quality for polluted rivers: an approach using pseudomeasurements; Trans. SICE, Vol. 43, No. 5, pp. 408417 (2007) (in Japanese)

[13] A. Ohsumi and S. Yasuki: Tracking of a maneuvering target considering its kinematic constraints; Intelligent Automation and Control-Recent Trends in Development and Applications, Vol. 9, (eds: M. Jamshidi, P. Borne and J. S. Jamshidi), TSI Press, pp. 407-416 (2000)

[14] A. Ohsumi, S. Yasuki, J. Hirata, Y. Ijiri, Y. Nagayama and H. Sasaki: Tracking of a maneuvering target with unknown course and speed using extended Kalman filter; Trans. ISCIE, Vol. 14, No. 10, pp. 490-498 (2001) (in Japanese)

[15] T. L. Song, J. Y. Ahn and C. Park: Suboptimal filter design with pseudomeasurements for target tracking; IEEE Trans. on Aerospace and Electric Systems, Vol. 24, No. 1, pp. 28-38 (1988)

[16] M. Tahk and J. L. Speyer: Target tracking problems subject to kinematic constraints; IEEE Trans. on Automatic Control, Vol. 35, No. 3, pp. 324-326 (1990)

[17] A. Tanikawa: Identification of unknown parameters of partially observed discrete-time stochastic systems by using a new class of pseudomeasurements (in preparation)

[18] A. Tanikawa and Y. Sawada: Identification of partially unknown system matrix of discrete-time stochastic systems via pseudomeasurement approach; Proc. of the 44th ISCIE Int. Symp. on Stochastic System Theory and Its Applications, pp. 107-112 (2012)

[19] P. Van Overschee and B. De Moor: N4SID: Subspace algorithms for the identification of combined deterministic-stochastic systems; Automatica, Vol. 30, No. 1, pp. 75-93 (1994)

[20] D. W. Whitecombe: Pseudo state measurements applied to recursive nonlinear filtering; Proc. of the 3rd Symp. on Estimation Theory and Its Appl., pp. 278$281(1972)$

\section{Appendix}

In Appendix, we discuss when the pair $\left(A_{u}, H\right)$ of the matrices $A_{u}$ and $H$ satisfies Condition A. We consider the simple case $(n, m, p)=(3,2,1)$. Let the matrix $H$ be given by one of the following specific forms for simplicity:

$$
H_{1}=\left[\begin{array}{lll}
1 & 0 & 0 \\
0 & 1 & 0
\end{array}\right], \quad H_{2}=\left[\begin{array}{lll}
0 & 1 & 0 \\
0 & 0 & 1
\end{array}\right], \quad H_{3}=\left[\begin{array}{lll}
1 & 0 & 0 \\
0 & 0 & 1
\end{array}\right] .
$$

Then, we immediately have

$$
\begin{aligned}
L_{1}:=H_{1}{ }^{\dagger} H_{1} & =\left[\begin{array}{lll}
1 & 0 & 0 \\
0 & 1 & 0 \\
0 & 0 & 0
\end{array}\right], L_{2}:=H_{2}{ }^{\dagger} H_{2}=\left[\begin{array}{lll}
0 & 0 & 0 \\
0 & 1 & 0 \\
0 & 0 & 1
\end{array}\right], \\
L_{3}:=H_{3}{ }^{\dagger} H_{3} & =\left[\begin{array}{lll}
1 & 0 & 0 \\
0 & 0 & 0 \\
0 & 0 & 1
\end{array}\right] .
\end{aligned}
$$

Noting that $p=1$, we denote the unknown parameter vector $a$ by $\alpha$ (a real number) and the unknown part of $A$ by $A_{u}(\alpha)$ in this section. Then, the matrix $A_{u}(\alpha)$ has the form

$$
\left(A_{u}(\alpha)\right)_{i j}= \begin{cases}\alpha & \left(i=i_{0}, j=j_{0}\right) \\ 0 & \left(i \neq i_{0} \text { or } j \neq j_{0}\right)\end{cases}
$$

for some $i_{0}\left(1 \leq i_{0} \leq 3\right)$ and $j_{0}\left(1 \leq j_{0} \leq 3\right)$. The pair $\left(i_{0}, j_{0}\right)$ is called the location of the unknown parameter $\alpha$ of the matrix $A_{u}(\alpha)$. For each location of $\alpha$ and each choice of $H$, we indicate whether the pair $\left(A_{u}, H\right)$ of the matrices $A_{u}$ and $H$ satisfies Condition $\mathrm{A}$ or not in the following table.

\begin{tabular}{|c|c|c|c|}
\hline the location of $\alpha$ & $H_{1}$ & $H_{2}$ & $H_{3}$ \\
\hline$(1,1)$ & $\mathrm{S}$ & $\mathrm{O}$ & $\mathrm{S}$ \\
\hline$(1,2)$ & $\mathrm{S}$ & $\mathrm{O}$ & $\mathrm{N}$ \\
\hline$(1,3)$ & $\mathrm{N}$ & $\mathrm{O}$ & $\mathrm{S}$ \\
\hline$(2,1)$ & $\mathrm{S}$ & $\mathrm{N}$ & $\mathrm{O}$ \\
\hline$(2,2)$ & $\mathrm{S}$ & $\mathrm{S}$ & $\mathrm{O}$ \\
\hline$(2,3)$ & $\mathrm{N}$ & $\mathrm{S}$ & $\mathrm{O}$ \\
\hline$(3,1)$ & $\mathrm{O}$ & $\mathrm{N}$ & $\mathrm{S}$ \\
\hline$(3,2)$ & $\mathrm{O}$ & $\mathrm{S}$ & $\mathrm{N}$ \\
\hline$(3,3)$ & $\mathrm{O}$ & $\mathrm{S}$ & $\mathrm{S}$ \\
\hline
\end{tabular}

In the table, " $\mathrm{S}$ " indicates that Condition $\mathrm{A}$ and $H_{p}(t)$ $\not \equiv O$ hold, whereas "O" indicates that Condition A holds but $L M_{0}=O$ (and so $H_{p}(t) \equiv O$ ). On the other hand, "N" indicates that Condition A does not hold. In view of the table, for any location of $\alpha$, we can find a matrix $H_{j}$ such that the pair $\left(A_{u}, H_{j}\right)$ satisfies Condition A and we can apply the pseudomeasurement approach to the system with $\left(A_{u}, H_{j}\right)$.

We now turn to the case: $p=2$. Let the unknown parameter vector $a$ be given by $a=\left[\alpha_{1}, \alpha_{2}\right]^{T}$. Using the notations $M_{i}\left(\alpha_{j}\right)$ for the matrix $M_{i}$ corresponding to $A_{u}\left(\alpha_{j}\right)$ and $M_{i}(a)=M_{i}\left(\alpha_{1}, \alpha_{2}\right)$ for the matrix $M_{i}$ corresponding to $A_{u}(a)$ respectively for $i=0,1,2,3$ and $j=1,2$, we immediately have

$$
\begin{aligned}
& M_{0}\left(\alpha_{1}, \alpha_{2}\right)=\left[M_{1}\left(\alpha_{1}\right) M_{1}\left(\alpha_{2}\right) M_{2}\left(\alpha_{1}\right) M_{2}\left(\alpha_{2}\right)\right. \\
& \left.M_{3}\left(\alpha_{1}\right) M_{3}\left(\alpha_{2}\right)\right] \text {. }
\end{aligned}
$$

Then, we can show the following lemma easily.

[Lemma 1] If for some $k(1 \leq k \leq 3)$ the equalities

$$
L_{k} M_{0}\left(\alpha_{j}\right)\left[\left(I-L_{k}\right) d\right]=0 \text { (the zero vector) }
$$

hold for $j=1,2$ and for any $d\left(\in \mathbb{R}^{3}\right)$, then we have 


$$
L_{k} M_{0}\left(\alpha_{1}, \alpha_{2}\right)\left[\left(I-L_{k}\right) d \otimes I_{2}\right]=O
$$

for any $d\left(\in \mathbb{R}^{3}\right)$.

(Proof) Writing $\left(I-L_{k}\right) d=\left[\tilde{d}^{(1)}, \tilde{d}^{(2)}, \tilde{d}^{(3)}\right]^{T}$, we have

$$
\begin{aligned}
& L_{k} M_{0}\left(\alpha_{1}, \alpha_{2}\right)\left[\left(I-L_{k}\right) d \otimes I_{2}\right] \\
& =L_{k}\left[M_{1}\left(\alpha_{1}\right) M_{1}\left(\alpha_{2}\right) M_{2}\left(\alpha_{1}\right) M_{2}\left(\alpha_{2}\right)\right. \\
& \left.M_{3}\left(\alpha_{1}\right) M_{3}\left(\alpha_{2}\right)\right]\left[\begin{array}{cc}
\tilde{d}^{(1)} & 0 \\
0 & \tilde{d}^{(1)} \\
\tilde{d}^{(2)} & 0 \\
0 & \tilde{d}^{(2)} \\
\tilde{d}^{(3)} & 0 \\
0 & \tilde{d}^{(3)}
\end{array}\right]
\end{aligned}
$$

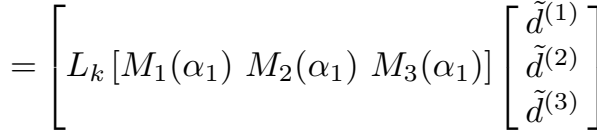

$$
\begin{aligned}
& \left.L_{k}\left[M_{1}\left(\alpha_{2}\right) M_{2}\left(\alpha_{2}\right) M_{3}\left(\alpha_{2}\right)\right]\left[\begin{array}{c}
\tilde{d}^{(1)} \\
\tilde{d}^{(2)} \\
\tilde{d}^{(3)}
\end{array}\right]\right] \\
& =\left[L_{k} M_{0}\left(\alpha_{1}\right)\left(I-L_{k}\right) d \quad L_{k} M_{0}\left(\alpha_{2}\right)\left(I-L_{k}\right) d\right] \\
& =\mathrm{O}
\end{aligned}
$$

When $a=\left[\alpha_{1}, \alpha_{2}, \cdots, \alpha_{p}\right]^{T}$ and $p \geq 3$, the notation $M_{i}(a)=M_{i}\left(\alpha_{1}, \alpha_{2}, \cdots, \alpha_{p}\right)$ is used for the matrix $M_{i}$ corresponding to $A_{u}(a)$ respectively for $i=0,1,2,3$. Lemma 1 can be easily extended to the case where $p \geq 3$. Thus, we obtain the following result.

[Corollary 4] If $a=\left[\alpha_{1}, \alpha_{2}, \cdots, \alpha_{p}\right]^{T}$ and $M_{0}\left(\alpha_{\ell}\right)$ satisfies (A1) for any $\ell(\ell=1,2, \cdots, p)$ for some $k(1 \leq k \leq$ $3)$, then $M_{0}(a)$ satisfies the equality

$$
L_{k} M_{0}\left(\alpha_{1}, \alpha_{2}, \cdots, \alpha_{p}\right)\left[\left(I-L_{k}\right) d \otimes I_{p}\right]=O
$$

for any $d\left(\in \mathbb{R}^{3}\right)$. Hence, the pair $\left(A_{u}(a), H_{k}\right)$ satisfies Condition A.

Choosing $p$ unknown parameters $\alpha_{\ell}(\ell=1,2, \cdots, p)$ whose locations indicate "S" for some $H_{k}$ in the table and putting $a=\left[\alpha_{1}, \alpha_{2}, \cdots, \alpha_{p}\right]^{T}$, we can obtain the system with the pair $\left(A_{u}(a), H_{k}\right)$ which satisfies Condition $\mathrm{A}$. We note that some of the indications at the locations of $\alpha_{\ell}$ 's can be "O" also.

\section{Authors}

Akio Tanikawa (Member)

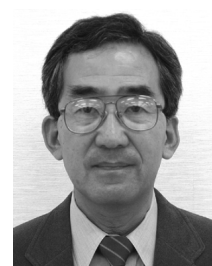

Received BS and MS in Mathematics, Nagoya University, Nagoya, Japan, in 1977 and 1979, respectively, and D.Sc. in Systems Science and Mathematics, Washington University, St. Louis, USA, in 1982. He was with Toyota College of Technology, Toyota, Japan from 1983 to 1988, with Kanazawa University, Kanazawa, Japan from 1988 to 1996, with Washington University, St. Louis, USA from 1996 to 2001, and with Oita University, Oita, Japan from 2001 to 2003. Since 2003 he has been Professor of Information Science, Osaka Institute of Technology, Osaka, Japan. His current research interests are in stochastic systems, system identification, optimal control, and game theory.

Yuichi SAWADA (Member)

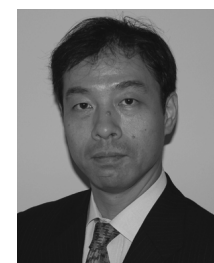

He received the M.Eng and D.Eng degrees in mechanical engineering from $\mathrm{Ky}$ oto Institute of Technology, Japan, in 1992 and 1994. He worked for Minolta Co., Ltd. from 1993 to 1994. He joined Department of Mechanical and System Engineering, Kyoto Institute of Technology as a Research Associate in 1995. Since 2006, Dr. Sawada has been an Associate Professor in Division of Mechanical and System Engineering at Kyoto Institute of Technology. He was a visiting Associate Professor of Department of Systems Science and Mathematics at Washington University in St. Louis, MO, USA from 1999 to 2000. His main research interests include control problems of mechanical systems, stochastic control design, nonlinear control systems, optimal filters and tele-operation systems. He is currently members of IEEE, IMechE, SICE, JSME, RSJ. 